\title{
猕猴毛发皮质醇测量方法探索
}

\author{
李春禄 $^{1,2,3}$, 秦冬冬 ${ }^{1,2,3}$, 冯晓丽 ${ }^{1,2,3}$, 张 波 $^{1,2,3}$, 杨上川 ${ }^{2}$, 胡新天 ${ }^{2, *}$, 马原野 ${ }^{1, *}$ \\ (1. 中国科学院昆明动物研究所 灵长类认知实验室, 动物模型和人类疾病机理重点实验室, 云南 昆明 650223; \\ 2. 中国科学院昆明动物研究所 灵长类感觉、运动及整合实验室, 动物模型和人类疾病机理重点实验室, 云南 昆明 650223 ;
}

3. 中国科学院研究生院, 北京 100049)

摘要: 毛发是评估下丘脑一垂体一肾上腺轴(HPA轴)慢性活性变化很好的样品, 毛发皮质醇的提取方法非常重 要。为保证毛发皮质醇提取过程的可靠性, 实验使用了液氮冷冻研磨; 设计了空白对照组（磷酸缓冲液(PBS)） $(n=3)$ 、原管组 $(n=10)$ 和新管组 $(n=10)$, 以检验缓冲液、离心管对提取的潜在干扰; 对同一动物样品 $(n=16)$ 两次 独立提取的结果及毛发皮质醇浓度与受到的极端攻击量（血浆皮质醇浓度的间接指标）( $n=16$ )进行了 Pearson相关 分析。结果表明: 空白对照组测量结果为 $0 \mathrm{pg} / \mathrm{mg}$; 新管浓度值与原管无显著性差异 $\left(F_{(1,19)}=0.041, P=0.843\right.$, one-way ANOVA); 两次独立提取结果高度正相关 $(r=0.893, P=0.000)$; 皮质醇浓度与受到的极端攻击量中度正相关 $(r=0.591, P=0.008)$ 。因此, 离心管、缓冲液对实验没有显著性干扰, 溶液中皮质醇来源于毛发样品; 提取操 作流程可靠, 所得数据可信。

关键词: 猕猴; 毛发; 皮质醇; 提取

中图分类号: Q959.848; Q513 文献标识码: A 文章编号: 0254-5853-(2009)04-0401-05

\section{A Reliable Procedure for Measuring Cortisol Levels in Rhesus Macaque Hair Samples}

\author{
LI Chun-lu ${ }^{1,2,3}$, QIN Dong-dong ${ }^{1,2,3}$, FENG Xiao-li1 ${ }^{1,2,3}$, Zhang Bo ${ }^{1,2,3}$, \\ YANG Shang-chuan ${ }^{2}$, HU Xin-tian ${ }^{2, *}$, MA Yuan-ye ${ }^{1, *}$ \\ (1. Laboratory of Primate Cognitive Neuroscience Research and Key Laboratory of Animal Models and Human Disease Mechanisms, Kunming Institute \\ of Zoology, the Chinese Academy of Sciences, Kunming 650223, China; 2. Laboratory of Sensory-motor Integration Research and Key Laboratory \\ of Animal Models and Human Disease Mechanisms, Kunming Institute of Zoology, the Chinese Academy of Sciences, Kunming 650223, China; \\ 3. Graduate School of the Chinese Academy of Sciences, Beijing 100049
}

\begin{abstract}
Hair provides a suitable sample inassessing long-term changes in hypothalamic-pituitary-adrenocortical axis (HPA) system activity. The procedure used for hair analysis is therefore very important. To ensure that our procedure is reliable, liquid nitrogen was used to deepfreeze the samples before powdering them. Experimental trials used three treatment types: blank group (phosphate buffered saline (PBS)) $(n=3)$, old tube group $(n=10)$ and new tube group $(n=10)$. The potential effects of PBS and tubes was then investigated. Additionally, both the relationship between the results of two samples of the same individuals $(n=16)$ yielded by the same procedures but at different times, and the relationship between hair cortisol concentrations and the number of received intense aggressions $(n=16)$ were also investigated. The results showed that the cortisol concentration of the blank group was $0 \mathrm{pg} / \mathrm{mg}$; new tubes did not differ from old tubes ( $F$ $(1,19)=0.041, P=0.843$, one-way ANOVA); and there was a high association between the results of two samples of the same individuals, yielded by same procedures operated at different times $(r=0.893, P=0.000)$. Moreover, hair cortisol concentrations were moderately correlated with the number of received intense aggressions $(r=0.591, P=0.008)$. Therefore, PBS and tubes did not affect the results significantly, therefore, our procedure was deemed reliable.
\end{abstract}

Key words: Rhesus Macaque; Hair; Cortisol; Extract 
糖皮质激素是一种应激激素, 可以调节糖、蛋 白质和脂肪的代谢, 提高机体对伤害性刺激的抵抗 力, 在动物生存中起到了相当重要的作用。皮质醇 是人类和非人灵长类糖皮质激素的主要成分 (Davenport et al, 2006)。因此, 皮质醇通常用作衡 量动物受到的应激刺激的大小的指标(Stavisky et al, 2001)。测量时样品一般使用血清或者血浆, 但 是这种样品对研究应激的实验来说却存在许多问 题, 例如: 取样过程中的捕捉、麻醉、取样前是否 进食、取样前短时间内环境变化引起的躁动(Puri et al, 1981; Creel et al, 1992; Davenport et al, 2006) 及皮质醇的昼夜节律变化(Rose et al, 1978; Plant, 1981; Umberkoman-Wiita et al, 1981) 都会对动物的 应激实验造成干扰。在动作敏捷、个体较大的群养 动物应激研究中, 问题更为突出: 如多次采血带来 的动物福利问题; 由于不能在短时间内捕捉到动 物, 捕捉时目标动物和同笼的其他动物所受到的应 激影响; 多次重复捕捉给动物带来的影响(Creel, 2001)。这些问题都可能会影响应激相关研究实验数 据的可信性(Creel, 2001), 阻碍了个体较大的群养 动物 (如狝猴一一种非常好的人类疾病研究模 型）的应激研究。因此, 取样技术的选择在应激研 究中显得尤其重要。

为了避免这些问题, 毛发样品是一种很好的选 择, 血液里的物质可以通过毛细血管渗透到毛囊细 胞, 最后扩散到毛发里(Cone, 1996)。因此, 从毛 发中可以提取出皮质醇。毛发样品有许多独特的优 点: (1) 毛发中的皮质醇是数周, 甚至数月的积 累总和, 不会受到昼夜节律的影响; (2) 可以避 免取样过程中捕捉、麻醉和其他因素带来的应激干 扰, 动物捕捉次数也明显减少; (3) 毛发易采集、 安全、方便储存和运输(Koren et al, 2002)。因此, 近两年国外已经有少量实验室将毛发皮质醇应用 于人和狝猴的相关研究, 并证实毛发分析是很好的 评估下丘脑-垂体-肾上腺(HPA)系统慢性活性变化 的工具(Davenport et al, 2006; Kalra et al, 2007; Yamada et al, 2007)。然而, 国内还没有类似实验 的报道。我们利用实验室现有条件, 在猕猴上对这 种方法进行了探索和改进, 希望能给国内研究者提 供参考, 推动国内的相关研究。

\section{1 材料和方法}

\section{1 实验动物}

实验使用的 16 只成年雄性猕猴 (Macaca mulatta), 年龄在 7 到 19 岁之间, 生活在 3 个 $3 \mathrm{~m} \times 5$ $\mathrm{m} \times 3 \mathrm{~m}$ 猴笼中 $(n=4,5,7)$ 。动物饲养在中科院昆 明动物研究所灵长类研究中心。除标准猴粮外, 每 天投喂水果或者蔬菜, 自由饮水。实验时, 动物均 已经生活在各自笼内至少 5 个月。

\section{2 行为观察}

有研究表明: 猕猴受到的极端攻击量可以预测 血浆皮质醇的水平, 它们具有正相关性(Gust et al, 1993)。因此, 我们对动物进行了行为观察, 用受到 的极端攻击量作为血浆皮质醇水平的一个衡量指 标。实验使用了追踪取样和行为取样(local sampling and behavior sampling)两种方法记录动物行为并进 行了录像。每天观察时间为: 上午 8: 40-9: 40; 9: 50-10: 50; 下午 14: 30-15：30；15：4016: 40; 每只动物一天观察 $2 \mathrm{~h}$, 共观察 $6 \mathrm{~h} 。 16$ 只动物共观察了 $96 \mathrm{~h}$ 。实验中记录了极端攻击行为: 咬、抓、打 (Shively et al, 2006)。

\section{3 取样过程}

15: 00-16: 00 期间, 将动物捕捉住后, 肌肉 注射氯胺酩 $(15 \mathrm{mg} / \mathrm{kg})$ 麻醉, 之后将动物移出笼 子。小心地用理毛器采集颈部后顶区的毛发, 约 5 $\mathrm{cm}^{2}$ 。用铝箔将毛发包好, 避免污染。

\section{4 毛发清洗和提取过程}

Davenport等 (2006) 对样品清洗过程做了详尽 研究, 我们就采用他们的清洗方法(Davenport et al, 2006)。样品粉碎是提取毛发皮质醇非常关键的一 步, 从相关文献中可以发现他们对这一步骤的改 进: 起初为剪碎(Koren et al, 2002), 后来两个实验 室使用了德国莱驰MM200 进行研磨粉碎(Raul et al, 2004; Davenport et al，2006)。我们使用了德国 莱驰MM400, 并在文献基础上做了一定改进: 将样 品放入液氮内充分冷冻变脆后, 再进行研磨, 这样 既可以使研磨粉末更细、更均匀, 又可以避免研磨 过程中局部高温对样品造成的影响。参考多个文献 后, 我们最终选择了甲醇(Koren et al, 2002; Davenport et al, 2006; Kalra et al, 2007; Yamada et al, 2007), 作为提取试剂, 其具体操作步骤: (1) 称取 $500 \mathrm{mg}$ 毛发样品置于 $15 \mathrm{~mL}$ 玻璃试管中, 加入 $10 \mathrm{~mL}$ 异丙醇。在室温下将该试管放于调速多用振 荡器上洗涤 $3 \mathrm{~min}$ (频率 100 次 $/ \mathrm{min}$ ), 共洗涤 2 次; 洗涤后的样品置于电热恒温干燥箱内, $37^{\circ} \mathrm{C}$ 干燥 8 $\mathrm{h}$ 。(2) 干燥后将样品放入 $80 \mathrm{~mL}$ 小烧杯内, 用手术 
剪剪碎。之后放入 $25 \mathrm{~mL}$ 不锈钢研磨罐内（内有 15 $\mathrm{mm}$ 不锈钢研磨球一个), 拧紧后将罐放入液氮内冷 冻至液氮停止沸腾。将研磨罐装入冷冻混合型球磨 仪MM400, 研磨 $2.5 \mathrm{~min}$ (频率 $26 \mathrm{~Hz}$ )。(3) 称取 $400 \mathrm{mg}$ 毛发粉末, 置于 $10 \mathrm{~mL}$ 离心管内, 并加入 $8 \mathrm{~mL}$ 甲醇。室温下, 将离心管置于振荡器, 振荡 $24 \mathrm{~h}$ (频 率 230 次 $/ \mathrm{min}$ ), 提取皮质醇。(4) 将离心管离心 5 $\min$ (离心力 $12000 \mathrm{~g}$, ), 取 $4 \mathrm{~mL}$ 上清液移入 $5 \mathrm{~mL}$ 离 心管内。(5) 在 $5 \mathrm{~mL}$ 离心管内取 $2 \mathrm{~mL}$ 上清液放入 2 $\mathrm{mL}$ 离心管, 室温用氮气流进行干燥, 样品干燥后, 加入 $5 \mathrm{~mL}$ 离心管内的剩余样品, 继续干燥 (随着甲 醇的挥发, 离心管内出现悬浮物。我们对这些悬浮 物进行了研究)。(6) 干燥后加入 $0.5 \mathrm{~mL}$ PBS (磷酸 缓冲液: $\mathrm{NaCl} 8 \mathrm{~g}, \mathrm{KCl} 0.2 \mathrm{~g}, \mathrm{Na}_{2} \mathrm{HPO}_{4} 1.44 \mathrm{~g}, \mathrm{KH}_{2}$ $\mathrm{PO}_{4} 0.24 \mathrm{~g}$, 加去离子水定容至 $1 \mathrm{~L}$, 将 $\mathrm{pH}$ 值调整到 $7.4)$ 对样品进行重构。振荡混匀后, 放入 $-20^{\circ} \mathrm{C}$ 冰箱 内储存至检测日。

\section{5 放免检测}

实验应用了美国进口Cortisol RIA DSL-2000 试 剂盒, 这种试剂盒主要用于血清、血浆和尿液皮质 醇的定量测量。我们使用了昆明医学院第二附属医 院自动放免仪, 按照试剂盒说明书进行了放免检 测。检验前将所有试剂在室温下平衡, 并充分混匀。 检验步骤如下: (1) 标记T管、标准品管、质控品 管和样品管，并按次序排列好; (2) 向相应管内 加入 $25 \mu \mathrm{L}$ 标准品、质控品、样品; (3) 向所有管 内加入 $500 \mu \mathrm{L}\left[\mathrm{I}^{-125}\right]$; (4) 彻底混匀抗血清, 向除 总 $\mathrm{T}$ 管外的所有管内加入 $500 \mu \mathrm{L}$ 抗血清; (5) 混匀, $37^{\circ} \mathrm{C}$ 水浴锅内温育 $45 \mathrm{~min}$;（6）将标准品管、质控 品管和样品管低温离心（1500g） $15 \mathrm{~min}$ （7）将 除总 $\mathrm{T}$ 管外的所有管上清液吸干; (8) 放入自动放 免仪计数, 并计算结果。为了数据统计需要, 我们 将数据单位从 $\mu \mathrm{g} / \mathrm{dL}$ 转换成了 $\mathrm{pg} / \mathrm{mg}$ 。

表 1 实验仪器和试剂

Tab. 1 Instrument and agent

\begin{tabular}{|c|c|c|c|c|c|}
\hline 仪器 Instrument & 型号 Type & $\begin{array}{c}\text { 生产厂家 } \\
\text { Manufacturer }\end{array}$ & 试剂 Agent & $\begin{array}{c}\text { 浓度 } \\
\text { Concentration }\end{array}$ & $\begin{array}{c}\text { 生产厂家 } \\
\text { Manufacturer }\end{array}$ \\
\hline $\begin{array}{l}\text { 调速多用振荡器 } \\
\text { Cycling shaker }\end{array}$ & HY-2 & $\begin{array}{l}\text { 江苏天由有限公司; 常州 } \\
\text { 国华电器有限公司 }\end{array}$ & $\begin{array}{l}\text { 甲醇 } \\
\text { Methanol }\end{array}$ & 99.5 & $\begin{array}{l}\text { 天津市风船化学试剂科技 } \\
\text { 有限公司 }\end{array}$ \\
\hline $\begin{array}{l}\text { 电热恒温干燥箱 } \\
\text { Drying stove }\end{array}$ & DHG-9011A & $\begin{array}{l}\text { 上海精宏实验设备有限 } \\
\text { 公司 }\end{array}$ & $\begin{array}{l}\text { 异丙醇 } \\
\text { Isopropanol }\end{array}$ & 99.7 & $\begin{array}{l}\text { 天津市风船化学试剂科技 } \\
\text { 有限公司 }\end{array}$ \\
\hline $\begin{array}{l}\text { 冷冻混合型球磨仪 } \\
\text { Retsch ball mill }\end{array}$ & MM400 & 德国莱驰公司 & $\begin{array}{l}\text { 氮气 } \\
\text { Nitrogen gas }\end{array}$ & 99.99 & 昆明天贝气体公司 \\
\hline 离心机 Centrifuge & H-1650 & $\begin{array}{l}\text { 湘仪离心机仪器 } \\
\text { 有限公司 }\end{array}$ & $\begin{array}{l}\text { 液氮 } \\
\text { Liquid nitrogen }\end{array}$ & & 昆明动物所中心实验室 \\
\hline $\begin{array}{l}\text { 自动放免仪 } \\
\text { Automatic } \\
\text { radioimmunoassay } \\
\text { assembly }\end{array}$ & GC-1200 & 中国科大中佳公司 & $\begin{array}{l}\text { 皮质醇放免 } \\
\text { 试剂盒 } \\
\text { Cortisol RIA }\end{array}$ & DSL-2000 & $\begin{array}{l}\text { 美国 Diagnostic } \\
\text { Systems Laboratories }\end{array}$ \\
\hline
\end{tabular}

\section{6 实验设计}

(1)为排除离心管和 PBS (磷酸缓冲液)带来的 假阳性, 我们将 $0.5 \mathrm{mLPBS}$ 放入离心管进行了放免 检测 $(n=3)$ 。(2)离心管壁可能会吸附皮质醇, 对皮质 醇浓度测量造成影响, 为检测这种影响, 我们将重 构后的样品的 $1 / 2$ 移入新管, 如果有吸附作用, 则 新管 $(n=10)$ 中样品由于经过了两次吸附, 皮质醇浓 度应低于原管 $(n=10)$ 。(3)为了检验悬浮物对测量结 果是否有影响, 我们对同一样品的浊液 $(n=10)$ 和清 液 $(n=10)$ 进行了比较。(4)为了保证实验操作的可靠 性, 我们对其中一批样品 $(n=16)$ 在不同时间进行了 两次放免测量。对同一只动物 $(n=16)$ 的两个样品,
不同时间做了同样的处理。(5)为了证明毛发中的皮 质醇可以反映动物受到的应激大小, 我们对毛发中 的皮质醇浓度与动物受到的极端攻击量 $(n=16)$ 进行 了相关分析。

\section{7 数据分析}

实验设计 (2)、(3) 的数据采用了 One-way ANOVA 分析。实验设计(4)、(5)数据采用了 Pearson correlation分析。所有分析均由SPSS 13.0 软件完成。 $P<0.05$ 为显著性差异, $P<0.01$ 为极显著性差异。 $|r|>0.95$ 显著性相关; $|r| \geq 0.8$ 高度相关; $0.5 \leq|r|$ $<0.8$ 中度相关; $0.3 \leq|r|<0.5$ 低度相关; $|r|<0.3$ 关系极弱, 认为不相关。 


\section{2 结 果}

实验结果表明, PBS测量结果为 $0 \mathrm{pg} / \mathrm{mg}$, 新管 浓度 值 $[(184.25 \pm 39.33) \mathrm{pg} / \mathrm{mg}]$ 与 原 管 [(196.38 \pm $45.56) \mathrm{pg} / \mathrm{mg}$ ]无显著性差异 $\left(F_{(1,19)}=0.041, P=0.843\right.$, one-way ANOVA)。浊液 [(167.50 19.44$) \mathrm{pg} / \mathrm{mg}$ 与与清 液 $(170 \pm 21.18) \mathrm{pg} / \mathrm{mg}$ ]无显著性差异 $\left(F_{(1,19)}=0.011\right.$, $P=0.918$, one-way ANOVA), 因此悬浮物对检测 结果没有明显影响。受到的极端攻击量与毛发皮质 醇浓度具有中度相关性 $(r=0.591, P=0.008$, Pearson correlation, 图 1)。同一样品两次测量结果呈现高度 相关性 $(r=0.939, P=0.000$, Pearson correlation, 图 $2 \mathrm{a})$ 。同一动物的两个样品皮质醇浓度也高度相关 ( $r=0.893, P=0.000$, Pearson correlation, 图 2b)。 更重要的是我们得到的猕猴毛发皮质醇浓度主要 集中在 $50 \sim 300 \mathrm{pg} / \mathrm{mg}$ 之间, 与狝猴文献数据(32.1 $254.3 \mathrm{pg} / \mathrm{mg}$ )(Davenport et al, 2006)接近。只有一只 动物毛发皮质醇浓度超过了 $400 \mathrm{pg} / \mathrm{mg}$, 但是这只

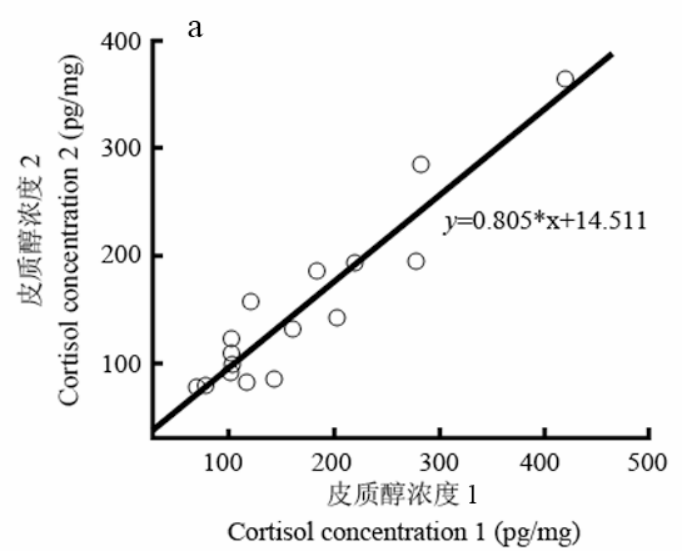

动物的两个样品浓度值是一致的。因此, 这可能是 由动物的个体差异造成的。

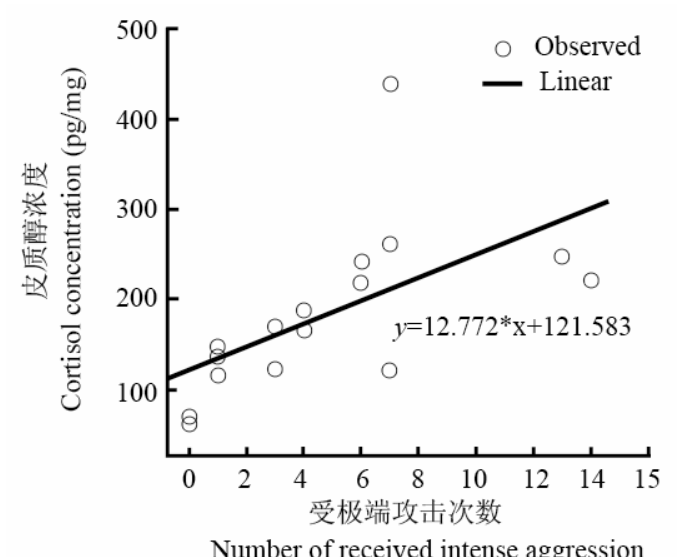

图 1 皮质醇浓度与受到的极端攻击次数相关性散点图 Fig. 1 Scatter plot illustrating the relationship between hair cortisol concentrations and the number of received intense aggressions across individual monkeys $(\mathrm{n}=16)$.

图 2 皮质醇浓度相关性散点图

Fig. 2 Scatter plot illustrating the relationship between the two cortisol concentrations, yielded by the same procedures operated at different times across individual monkeys $(n=16)$

$\mathrm{a}$ : 同一样品两次测量; $\mathrm{b}$ : 同一动物两个样品。

a: Results of the same sample; b: Results of two samples of the same individuals.

\section{3 讨 论}

整个提取过程中, 离心管和 PBS 并没有对样品 造成假阳性。这说明样品皮质醇来源于毛发, 而不 是污染。离心管壁没有吸附皮质醇, 因此不会对数 据的可靠性造成影响。重构后样品是一种浊液, 难 以分离, 不过我们实验证明样品里的悬浮物对结果 不会造成影响, 不需要分离。然而, 我们建议检测 前将样品混匀。同一样品两次测量的结果, 其高度
相关性表明了放免检测操作过程稳定，重复性好， 测量结果可信。同一动物两个样品间的高度相关性 为我们整个实验操作的可靠性提供了证据, 同时也 说明同一动物邻近毛发的皮质醇含量高度相关。受 到的极端攻击量虽然是血浆中皮质醇浓度的间接 指标, 但是它与毛发皮质醇浓度的中度相关性, 以 及我们实验数据与文献数据皮质醇含量范围的一 致性(Davenport et al, 2006), 都说明了我们确实提 取到了毛发中的皮质醇，数据可信，同时毛发皮质 
醇可以作为衡量应激刺激大小的指标。由我们的经 验及测量得到的样品皮质醇含量最低值和试剂盒 的灵敏度大致可以推算出: 在狝猴毛发皮质醇提取 实验中, 为得到足够浓度, 毛发样品量不宜低于 200 $\mathrm{mg}$ 。

\section{参考文献:}

Cone EJ. 1996. Mechanisms of drug incorporation into hair [J]. Ther Drug Mon, 18(4): 438.

Creel S. 2001. Social dominance and stress hormones[J]. Trends Ecol Evol, 16(9): 491-497.

Creel S, Creel N, Wildt DE, Monfort SL. 1992. Behavioural and endocrine mechanisms of reproductive suppression in Serengeti dwarf mongooses[J]. Anim Behav, 43: 231-245.

Davenport MD, Tiefenbacher S, Lutz CK, Novak MA, Meyer JS. 2006. Analysis of endogenous cortisol concentrations in the hair of rhesus macaques[J]. Gen Comp Endocrinol, 147(3): 255-261.

Gust DA, Gordon TP, Hambright MK, Wilson ME. 1993. Relationship between social factors and pituitary - adrenocortical activity in female rhesus monkeys (Macaca mulatta)[J]. Horm Behav, 27(3): 318-331.

Kalra S, Einarson A, Karaskov T, Van Uum S, Koren G. 2007. The relationship between stress and hair cortisol in healthy pregnant women[J]. Clin Invest Med, 30(2): E103.

Koren L, Mokady O, Karaskov T, Klein J, Koren G, Geffen E. 2002. A novel method using hair for determining hormonal levels in wildlife[J]. Anim Behav, 63(2): 403-406.

Plant TM. 1981. Time courses of concentrations of circulating gonadotropin, prolactin, testosterone, and cortisol in adult male rhesus monkeys (Macaca mulatta) throughout the 24h light-dark cycle[J]. Biol Reprod, 25(2): 244-252.

Puri CP, Puri V, Kumar TC. 1981. Serum levels of testosterone, cortisol, prolactin and bioactive luteinizing hormone in adult male rhesus monkeys
总结: 毛发皮质醇浓度可以作为衡量慢性应激 刺激大小的指标。我们实验室实现了国外同行所做 的提取过程，并做了一定的改进，提取过程真实可 靠。希望我们的工作能对从事应激相关研究的国内 同行有所帮助。

following cage-restraint or anaesthetizing with ketamine hydrochloride $[\mathrm{J}]$. Eur J Endocrinol, 97(1): 118.

Raul JS, Cirimele V, Ludes B, Kintz P. 2004. Detection of physiological concentrations of cortisol and cortisone in human hair[J]. Clin Biochem, 37(12): 1105-1111.

Rose RM, Gordon TP, Bernstein IS. 1978. Diurnal variation in plasma testosterone and cortisol in rhesus monkeys living in social groups[J]. $J$ Endocrinol, 76(1): 67-74.

Shively CA, Friedman DP, Gage HD, Bounds MC, Brown-Proctor C, Blair JB, Henderson JA, Smith MA, Buchheimer N. 2006. Behavioral depression and positron emission tomography-determined serotonin $1 \mathrm{~A}$ receptor binding potential in cynomolgus monkeys[J]. Arch Gen Psychiatry, 63: 396-403.

Stavisky RC, Adams MR, Watson SL, Kaplan JR. 2001. Dominance, cortisol, and behavior in small groups of female cynomolgus monkeys (Macaca fascicularis)[J]. Horm Behav, 39(3): 232-238.

Umberkoman-Wiita B, Hansen S, Herbert J, Moore GF. 1981. Circadian rhythms in serum and CSF cortisol of rhesus monkeys, and their modulation by timed injections of L-5-hydroxytryptophan[J]. Brain Res, 222(2): 235

Yamada J, Stevens B, de Silva N, Gibbins S, Beyene J, Taddio A, Newman C, Koren G. 2007. Hair cortisol as a potential biologic marker of chronic stress in hospitalized neonates[J]. Neonatology, 92(1): 42-49. 